\title{
Efficacy of an artificial tear emulsion in patients with dry eye associated with meibomian gland dysfunction
}

This article was published in the following Dove Press journal:

Clinical Ophthalmology

26 August 2013

Number of times this article has been viewed

\author{
Christine W Sindt \\ Gary N Foulks² \\ 'Department of Ophthalmology and \\ Visual Sciences, University of lowa \\ Hospitals and Clinics, lowa City, \\ IA, ${ }^{2}$ Kentucky Lions Eye Center, \\ Department of Ophthalmology and \\ Visual Science, University of Louisville, \\ Louisville, KY, USA
}

Objectives: The aim of the study reported here was to assess the efficacy of an artificial tear emulsion for the treatment of dry eye associated with meibomian gland dysfunction (MGD).

Methods: At five clinics, patients completed a 1-week treatment with their habitual topical therapy and then a 4-week treatment with open-label study medication: Systane ${ }^{\circledR}$ Balance Lubricant Eye Drops (Alcon, Alcon Inc, Fort Worth, TX, USA). Subjective assessments included a preference survey, the Impact of Dry Eye in Everyday Life questionnaire, and the Work Productivity and Activity Impairment questionnaire. Objective assessments by unmasked investigators included visual acuity, meibomian gland expression and dropout, tear film breakup time, corneal staining, and dosing frequency.

Results: At baseline, the 49 patients had mean meibomian gland expression grades and gland dropout that indicated mild to moderate MGD. Patients administered their habitual therapy $2.5 \pm 1.3$ times per day. After 4 weeks of study medication, the Impact of Dry Eye in Everyday Life questionnaire results indicated statistically and clinically significant improvements. Fewer than half of the participants were employed, limiting the usefulness of the Work Productivity and Activity Impairment questionnaire. Visual acuity remained statistically similar, while corneal staining and tear film breakup time improved significantly $(P<0.05)$ but modestly. The outcomes were achieved with $1.9 \pm 1.1$ doses per day of study medication, a significantly lower frequency than the habitual frequency $(P<0.001)$. The most common medication-related adverse event was blurred vision (3/49 patients, 6.1\%). At study conclusion, 27/44 (61.4\%) survey respondents preferred the study medication to their habitual therapy.

Conclusion: The artificial tear emulsion was effective for treating the signs and symptoms of dry eye in MGD patients.

Keywords: ophthalmic solutions, Work Productivity and Activity Impairment, Impact on Dry Eye in Everyday Life, MGD, tear film breakup time, corneal staining

\section{Introduction}

The two major classes of dry eye are aqueous-deficient dry eye and evaporative dry eye, which are not mutually exclusive. ${ }^{1}$ An intrinsic cause of evaporative dry eye is low-delivery meibomian gland dysfunction (MGD), ${ }^{1}$ which is one type of posterior blepharitis, ${ }^{2}$ and which can be further subcategorized as hyposecretory or obstructive MGD. ${ }^{2}$ Low-delivery MGD is more common than high-delivery (hypersecretory) $\mathrm{MGD}^{2}{ }^{2}$ with the former so prevalent that it is likely to be the most common cause of evaporative dry eye ${ }^{1}$ and is responsible for approximately $40 \%-60 \%$ of dry eye cases. $^{3-5}$ The widespread and problematic nature of MGD made it the topic of a recent international workshop of experts. ${ }^{6,7}$
Correspondence: Christine W Sindt Contact Lens Service, Department of Ophthalmology and Visual Science, University of lowa, 200 Hawkins Dr, lowa City, IA 52242-I09I, USA

Tel +l 3193564816

Fax +I 3193560363

Email christine-sindt@uiowa.edu 
Low-delivery MGD can be caused by a variety of combinations of changes to the number, structure, function, and flora of the glands, resulting in reduced or altered secretion of the oily meibum. ${ }^{2}$ It is not always clear how the sometimes clinically subtle signs in the glands and meibum can be associated with the occasionally extremely bothersome symptoms that are reported by patients. For dry eye in general, the poor correlation between dry eye assessments and dry eye symptoms has presented a quandary in clinical research and practice. ${ }^{8}$ For MGD in particular, one study found no association between meibomian gland grading and any of the following ocular symptoms, individually: dryness, grittiness, soreness, redness, and fatigue. ${ }^{8}$ Lipid layer thickness has been found to correlate to the symptoms of dry eye, ${ }^{9}$ but lipid layer thickness and composition are not easily measured with routine clinical instrumentation.

Treatment recommendations depend on the type and severity of MGD. ${ }^{10}$ For mild to moderate MGD, a recent treatment algorithm includes recommendations such as eyelid therapy (hygiene, heat, and massage), dietary omega-3 essential fatty acids, artificial lubricants, ophthalmic antibiotics, and ophthalmic anti-inflammatories. ${ }^{10}$ In 2010, a new nonprescription option became available: Systane ${ }^{\circledR}$ Balance Lubricant Eye Drops (Alcon Inc, Fort Worth, TX, USA). These drops are indicated for temporary relief of burning and irritation due to dryness of the eye. In particular, the formulation ${ }^{11}$ was expected to be beneficial for dry eye symptoms in MGD patients.

For a treatment to be considered successful for dry eye in MGD, it should alleviate both the symptoms and the signs of dry eye in MGD, in a manner that is compatible with the patient's lifestyle. The study reported here focused on subjective patient experiences by using three questionnaires to assess patient-reported outcomes and by setting the primary efficacy outcome of patient's preference for treatment (the habitual previous topical therapy versus the new study medication). In addition to the subjective outcomes, objective assessments included daily drop frequency, meibomian gland expression and dropout, corneal staining, tear film breakup time, and visual acuity. Adverse events were recorded for assessment of safety outcomes. Altogether, this comprehensive evaluation was designed to give a definitive assessment of the effect of this product for patients with dry eye associated with MGD.

\section{Materials and methods Administrative issues}

The study was conducted at five clinical sites in the United States, with each clinic targeting enrollment of 5 to 10 patients.
The protocol was approved by an institutional review board (IntegReview Ethical Review Board, Austin, TX, USA) and the study was registered at ClinicalTrials.gov as NCT01079858. ${ }^{12}$ Eligible participants were adults ( $\geq 18$ years old) who: provided written informed consent as a prerequisite for study participation; were willing and able to follow study instructions and maintain the appointment schedule; were not members of the investigators' office staff, family, or household; and had not participated in any other ophthalmic clinical trial within 30 days of the enrollment visit.

\section{MGD eligibility}

Eligible patients were required to have a diagnosis of dry eye associated with MGD. This diagnosis was defined as experiencing the symptoms of dry eye (itching, tearing, burning, and episodes of blurred vision) and exhibiting the signs of MGD on a gland expression examination. Gland expression was performed while the clinician examined the eyes using a slit lamp under $10 \times$ to $16 \times$ magnification. A cotton-tipped wooden applicator was applied for 5 to 10 seconds for expression, moving across the full lower lid from temporal to nasal. Meibum was graded as the global average of all expressions for one lid. Color and viscosity of meibum were graded using a previously established scale ${ }^{13}$ on which "grade 0 " represents normal, clear oil; "grade 1" represents opaque, diffusely turbid, normal viscosity oil; "grade 2" represents opaque, increased viscosity oil; and "grade 3" represents inspissated oil (ie, like toothpaste) or indicates that no material could be expressed. Eligible patients had a grade of 1 or worse in both eyes.

\section{Additional eligibility criteria}

Additional ocular criteria for inclusion in the study were a corrected distance visual acuity of 0.6 logarithm of the Minimum Angle of Resolution ( $\log$ MAR) or better in each eye at the enrollment visit and a history of an ocular examination in the 2 years preceding the enrollment visit. Participants were required to be using topical ocular dry eye therapy (artificial tears or cyclosporine ophthalmic emulsion, $0.05 \%$ [Restasis ${ }^{\circledR}$ prescription eye drops; Allergan, Irvine, CA, USA]) at least two times per day. Contact lens wearers who wished to participate in the study had to be willing to discontinue using contact lenses for the duration the study.

Ocular criteria that would cause exclusion from the study were: a history of ocular surgery or trauma within the past 6 months; the presence of punctal plugs that were placed within the 30 days prior to the enrollment visit; the presence of ocular infections, conjunctival abnormalities, eyelid 
abnormalities, or any other ocular condition that could, in the opinion of the investigators, preclude the safe administration of the test article; and a history or presence of a defined list of ocular inflammations and conditions of viral, bacterial, or fungal origin.

Patients who were taking a chronic systemic medication were required to have no changes to dosing within the 7 days prior to the enrollment visit and no changes to dosing planned during the duration of the study. Patients were ineligible if they had a history of systemic or ocular hypersensitivity to any component of the study medication.

\section{Objective baseline examination}

In addition to the visual acuity assessments and gland expression grading that were conducted for the eligibility screening, objective evaluations included assessments of meibomian gland dropout, tear film breakup time, and corneal staining. Assessment of meibomian gland dropout was conducted using a slit lamp, with the eyelid everted. Partially missing glands were assigned a value of $1 / 2$ (ie, 0.5$)$ dropout and absent glands were assigned a value of 1 dropout. Total glands missing were summed along the entire lower eyelid. Measurement of tear film breakup time was conducted by instilling $5 \mu \mathrm{L}$ of $2 \%$ preservative-free sodium fluorescein; after fluorescein was distributed by three blinks, a measurement was made while the clinician observed the eye through a cobalt filter using the slit lamp. Using a stopwatch, two tear film breakup times were recorded per eye, by measuring the time from the last blink until one or more black (dry) spots appeared in the precorneal tear film, as recommended in the Dry Eye Workshop Report of 2007. ${ }^{1}$ Thereafter, corneal fluorescein staining was graded for each of the five regions (central, nasal, temporal, superior, inferior) of each eye, on a scale ranging from 0 (no staining) to 3 (severe staining), as previously described. ${ }^{14}$

\section{Subjective baseline examination}

Participants completed a product preference questionnaire concerning their current habitual therapy (artificial tears or cyclosporine ophthalmic emulsion, $0.05 \%$ ). The product preference questionnaire was not a validated instrument but was designed for the purposes of this study. At baseline, this questionnaire had three questions to assess satisfaction with therapy and seven questions to assess perceptions of response to therapy.

Participants also completed a further two questionnaires. One was the validated Impact on Dry Eye in Everyday Life (IDEEL) questionnaire. ${ }^{15,16}$ The other was the Work Productivity and Activity Impairment: Specific Health Problem
(WPAI:SHP) questionnaire, version 2.0, which was validated in $1993^{17}$ and has been applied to a large variety of systemic and nasal disorders (as partially listed elsewhere) ${ }^{18}$ as well as to dry eye disease. ${ }^{19}$

\section{Dispensation to week I of the study}

After the successful completion of the enrollment visit, patients were instructed to continue using their habitual therapy according to their normal schedule for 7 days. Each patient was given an electronic medication monitoring device (Medication Event Monitoring System [MEMS] 6 SmartCap; Aardex, Union City, CA, USA), which is designed as a medication container. This device has been very widely used for decades in studies of oral medications ${ }^{20}$ and has been used for studies of ophthalmic medications. ${ }^{21,22}$ Patients were instructed to place their bottle of habitual eye drops into the MEMS container and they were informed orally and in writing that the MEMS container would track their dosing every time the patients opened the MEMS container cap to administer their eye drops. They were also advised that the MEMS device would electronically record the date and time of the opening, yielding records for all containercap openings in each 24-hour period.

\section{Visit 2 (medication change)}

After $7 \pm 2$ days of using their habitual dry eye therapy, patients returned for visit 2 of the study. Any changes in medication and any adverse events were recorded. Dosing devices were checked for compliance. Tear film breakup time and corneal staining measurements were undertaken. Thereafter, patients were instructed to discontinue using their habitual drops. Patients were given an open-label, 2-week supply of the study medication (Systane Balance Lubricant Eye Drops), and were instructed to use the dosing tracking device for the study medication. No washout period occurred between previous therapy and study medication, in accordance with immediate comparison, real-world scenarios.

\section{Visit 3 (interim assessment)}

After 2 weeks of using the new study medication (ie, after $21 \pm 2$ days of participating in the study), patients returned for study visit 3 . As at visit 2, assessments of medications, adverse events, dosing device compliance, tear film breakup time, and corneal staining were conducted. In addition, patients completed the three satisfaction questions of the patient preference survey, this time with respect to the study medication. Patients returned their previous supply of the study medication, were given a new 2-week supply, and were reminded to continue using the tracking device. 


\section{Visit 4 (outcomes assessment)}

After 2 further weeks of using the study medication (4 weeks total using the study medication, and 5 weeks total participating in the study), patients returned to the clinics for outcome assessments. Objective efficacy assessments were visual acuity, tear film breakup time, corneal staining, meibomian gland expression, and meibomian gland dropout. Subjective efficacy outcomes were the same three surveys as at baseline (WPAI:SHP, IDEEL, and patient preference); in addition, a new survey question asked patients whether they preferred their previous habitual therapy or the new study therapy. The question was worded as follows: "If the price of your previous eye drop and the new eye drop were equal, which would you choose? (check one only)," and response options were "previous medication" or "new medication."

Any changes in medication and any adverse events were recorded. The dosing devices and study medication were collected.

\section{Analyses of outcomes}

The results of the 57 questions on the IDEEL questionnaire were calculated for their previously established three modules and six subscales. ${ }^{15}$ In the quality-of-life module (with its three subscales of activity limitations, emotional well-being, and work impact), scores range from 0 (worst) to 100 (best). ${ }^{15}$ In the treatment satisfaction module (with its two subscales of treatment satisfaction and treatment bother), scores range from 0 (least satisfied) to 100 (most satisfied).${ }^{15}$ In the symptom bother module (one module, one subscale), scores range from 0 (least bother) to 100 (worst bother), ${ }^{15}$ where the minimum clinically important difference in symptom bother is twelve units of change. ${ }^{16}$ Effect size indices, as measures of clinical significance, were calculated as the change in mean scores divided by the baseline standard deviation. ${ }^{23} \mathrm{~A}$ small effect size is 0.2 to $<0.5$ units, a moderate effect size is 0.5 to $<0.8$ units, and a large effect size is $\geq 0.8$ units. ${ }^{23}$

As previously described for the WPAI:SHP questionnaire, ${ }^{17}$ work time missed or compromised because of dry eye was calculated as past-week percentage of presenteeism, absenteeism, and overall impairment (presenteeism plus absenteeism) for patients who held employment positions during the study. Also as previously described, ${ }^{17}$ the effect of dry eye on non-work activities (such as housework, exercising, and studying) was calculated as percentage impairment.

The primary objective was assessed as the overall product preference among the participants, defined as the percentage of subjects who preferred the test eye drops to their habitual eye drops after completing the study. Other outcomes on the patient preference survey were evaluated using the per-patient changes from baseline to week 5. For numeric variables such as dosing frequency, visual acuity, tear film breakup time, corneal staining scores, and WPAI:SHP and IDEEL scores, the paired $t$-test was used for per-patient before and after comparisons, and mean percent changes were calculated. For numeric variables that were collected at the level of the eye (including visual acuity, corneal staining, and gland expression), average-eye values were calculated per patient before calculating before and after comparisons. Analyses were performed using SAS (SAS Institute, Cary, NC, USA) and a confidence level of $95 \%$ for all tests (Numeric variables reported as mean \pm standard deviation $[\mathrm{SD}])$.

\section{Results \\ Demographics and follow-up}

A total of 49 patients were enrolled, with demographics as shown in Table 1. Previous ocular topical therapies were reported as monotherapies and as combination therapies; the most common component of a treatment regimen was lubricant eye drops or artificial tears, as shown in Table 1. For patients who were using combination therapies, the artificial tears or cyclosporine solution that the patients were using at least two times per day (as specified in the study protocol) was the bottle that was placed in the dosing tracking container

Table I Demographics and previous ocular topical therapies of the study population

\begin{tabular}{ll}
\hline Demographic/therapy & $\mathbf{n}(\%)$ \\
\hline Sex & \\
Men & $7(14.3)$ \\
Women & $42(85.7)$ \\
Age, years & \\
I8-39 & $8(16.3)$ \\
$40-59$ & $18(36.7)$ \\
$60-79$ & $18(36.7)$ \\
$\geq 80$ & $5(10.2)$ \\
Ocular topical therapy & \\
Lubricant drops/artificial tears & $41(83.7)$ \\
Cyclosporine emulsion & $8(16.3)$ \\
Emollient lubricant eye drops & $4(8.2)$ \\
Lubricant eye gel or gel drops & $4(8.2)$ \\
Lubricant eye ointment & $2(4.1)$ \\
Antihistamine eye drops & $3(6.1)$ \\
Homeopathic sterile eye drops & $1(2.0)$ \\
Uncertain classification & ${ }^{\mathrm{b}}$ \\
Brand of ocular topical therapy & $3(6.1)$ \\
Systane ${ }^{\circledR c}$ & \\
Not Systane & $23(46.9)$ \\
\hline
\end{tabular}

Notes: a Combinations are reported as separate components, so total is $>100 \%$; bsome brand names reported by the patients could have indicated lubricant drops, gels, or ointments; 'Alcon Inc, Fort Worth, TX, USA. 
for the first week of the study. Of the 49 patients who were enrolled, 48 patients completed the study. Not all outcomes were assessed for all patients (eg, some forms were returned with incomplete portions). Numbers of eyes or patients are specified where applicable.

\section{Subjective efficacy outcomes}

All six of the IDEEL subscales indicated that the patients experienced statistically and clinically significant improvements, as shown in Table 2 (raw values) and Figure 1 (relative changes). The mean IDEEL Symptom Bother module/subscale improvement was 14 units, exceeding the clinically important difference threshold of twelve units. The WPAI:SHP questionnaire results (Table 3) indicated no significant changes to absenteeism, presenteeism, and the combination of both; however, less than half of the study population was employed, resulting in low power to detect statistical differences in work-related parameters. For WPAI:SHP assessment of impairment to non-work activities, improvement was statistically significant $(P=0.006)$, with a mean magnitude of $11.3 \% \pm 26.4 \%$ alleviation of impairment.

For the primary study endpoint of patients' treatment preference, $61.4 \%(27 / 44)$ of patients responded that they would choose the new study medication, while $38.6 \%(17 / 44)$ responded that they would choose their previous medication. Among patients who had any type of "earlier-generation"

Table 2 Scores on the subscales of the Impact on Dry Eye in Everyday Life questionnaire at baseline (with habitual drops) and at visit 4 (after 4 weeks of treatment with study drops)

\begin{tabular}{|c|c|c|c|c|}
\hline \multirow[t]{2}{*}{ Module } & \multicolumn{2}{|c|}{$\begin{array}{l}\text { Baseline value } \\
\text { with habitual } \\
\text { drops }\end{array}$} & \multicolumn{2}{|c|}{$\begin{array}{l}\text { After } 4 \text { weeks } \\
\text { with study drops }\end{array}$} \\
\hline & Mean \pm SD & $\mathbf{n}$ & Mean \pm SD & $\mathbf{n}$ \\
\hline \multicolumn{5}{|l|}{ Quality-of-life module } \\
\hline $\begin{array}{l}\text { Daily activity limitations } \\
(100=\text { least limitation })\end{array}$ & $74 \pm 18$ & 49 & $81 \pm 19$ & 47 \\
\hline $\begin{array}{l}\text { Emotional well-being } \\
\text { (100= best well-being) }\end{array}$ & $74 \pm 20$ & 49 & $83 \pm 20$ & 47 \\
\hline $\begin{array}{l}\text { Work limitations } \\
(100=\text { least limitation })\end{array}$ & $73 \pm 22$ & $25^{\mathrm{a}}$ & $84 \pm 17$ & $23^{a}$ \\
\hline \multicolumn{5}{|c|}{ Treatment satisfaction module } \\
\hline $\begin{array}{l}\text { Treatment satisfaction } \\
(100=\text { most satisfied })\end{array}$ & $41 \pm 19$ & 49 & $55 \pm 28$ & 46 \\
\hline $\begin{array}{l}\text { Treatment bother } \\
(100=\text { least bother })\end{array}$ & $63 \pm 21$ & 49 & $79 \pm 18$ & 47 \\
\hline $\begin{array}{l}\text { Symptom bother module } \\
(100=\text { most bother })\end{array}$ & $58 \pm 18$ & 49 & $44 \pm 22$ & 47 \\
\hline
\end{tabular}

Notes: Each subscale ranges from 0 to 100. aPatients who were not employed did not complete the work limitations section.

Abbreviation: SD, standard deviation.
Systane product in their prior therapies, the newer-generation Systane Balance study medication was preferred by $70 \%$ $(14 / 23)$ of patients. Among patients who did not have any type of Systane product in their prior therapies $(n=26)$, the study medication was preferred by $54.2 \%(13 / 26)$ of patients. This difference (70\% versus $54.2 \%)$ was not investigated statistically but was conducted due to the open-label nature of the study and possible brand loyalties.

Additional assessments of the study medication, from the same preference survey, are shown in Table 4. For all seven questions, a majority of patients ( $>53 \%$ ) agreed or strongly agreed with favorable impressions of the study eye drops. Satisfaction with aspects of the study medication, from the same survey, are shown in Table 5. For all three questions, a majority of patients $(>78 \%)$ was somewhat satisfied or very satisfied with the aspects of the study medication.

\section{Objective efficacy outcomes}

With habitual therapy, study participants $(n=46)$ self-administered $2.5 \pm 1.3$ doses per day. With the study medication, that frequency decreased to $1.9 \pm 1.1$ doses per day. The paired mean decrease in dosing frequency was significant $(P<0.001)$, with a magnitude of $0.6 \pm 0.9$ fewer doses per day (see Figure 2).

Baseline and outcome mean meibomian gland expression grades were between grade 1 (opaque and turbid with normal viscosity) and grade 2 (opaque with increased viscosity), indicating mild to moderate dysfunction of meibomian gland expression. Specifically, values for expression grades were $1.6 \pm 0.7$ at baseline $(n=49$ patients $)$ and $1.2 \pm 0.6$ at visit $4(\mathrm{n}=47$ patients). Paired change from baseline was $-0.4 \pm 0.8$ grading units, or $-17 \% \pm 36 \%(n=47$ patients; $P=0.002)$.

At baseline, the mean number of glands that were missing was $5.5 \pm 4.3$ per eye $(n=49$ patients $)$. At visit 4 , meibomian gland dropout was statistically similar to baseline, as might be expected. The magnitude of change was $0.1 \pm 1.3$ glands ( $P=0.45$ versus baseline), at $5.6 \pm 4.4$ glands missing per eye $(n=47$ patients).

Changes in tear film breakup time from baseline to visit 4 were clinically modest but statistically significant, as shown in Table 6. Mean staining left behind after the tear film test was in the mild range for all corneal sectors, at baseline and at visit 4 , as shown in Table 7. Improvement in staining was significant for each corneal sector (all sectors, $P<0.05$ ) and for overall corneal score $(P<0.001)$, with a magnitude of $-1.0 \pm 1.3$ units of overall improvement (on a 16-point scale) per patient. 


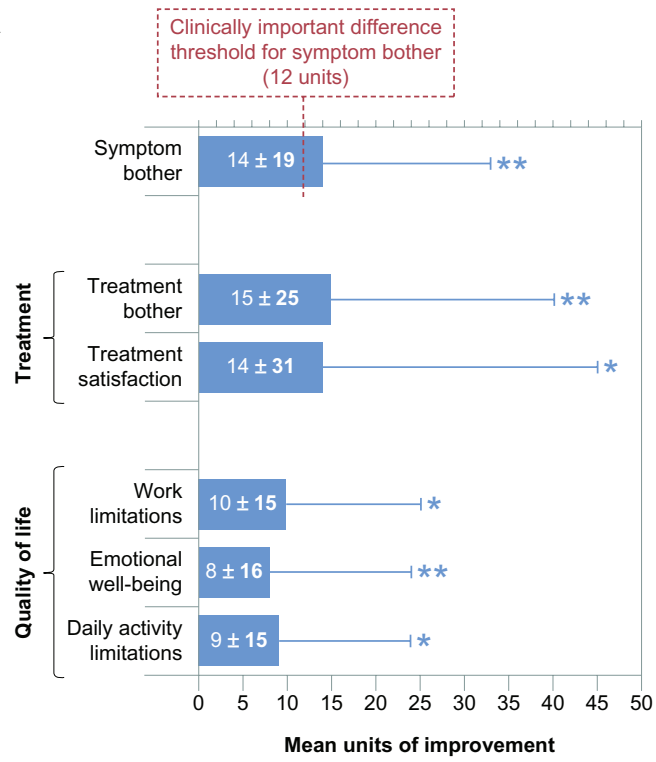

B

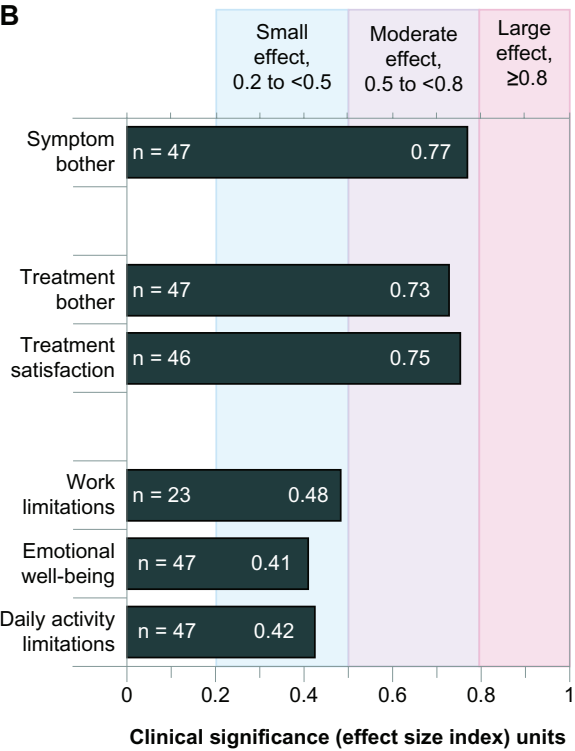

Figure I Improvement on the subscale scores of the Impact on Dry Eye in Everyday Life questionnaire, from baseline (with habitual drops) to visit 4 (after 4 weeks of treatment with study drops). (A) Mean change per patient; maximum possible change was 100 units. (B) Clinical significance of improvement (effect size indices). Notes: Error bars represent the standard deviation and are shown unidirectional for clarity. $* P<0.0$ I; $* * P<0.00$ I.

Baseline mean monocular corrected distance visual acuity was $0.07 \pm 0.16 \log \mathrm{MAR}(\mathrm{n}=49$ patients $)$. At visit 4 , mean visual acuity was $0.04 \pm 0.16 \log \operatorname{MAR}(\mathrm{n}=46)$. The per-patient improvement in visual acuity was $-0.03 \pm 0.11$ $\log$ MAR units $(P=0.08 ; \mathrm{n}=46)$.

\section{Adverse events}

Four patients reported six adverse events that were judged to be related to the study medication. The most common adverse

Table 3 Results of the Work Productivity and Activity Impairment Questionnaire analyses

\begin{tabular}{llll}
\hline Impairment due to dry eye & $\mathbf{n}$ & Mean \pm SD & $\mathbf{P}$ \\
\hline Work activity & & & \\
Absenteeism, \% of work time & & & \\
$\quad$ Baseline, with habitual drops & 22 & $1.4 \pm 5.8$ & \\
$\quad$ After 4 weeks with study drops & 19 & $0.1 \pm 0.5$ & \\
$\quad$ Change from baseline & $18^{\mathrm{a}}$ & $0.1 \pm 0.5$ & 0.330 \\
Presenteeism, \% impairment & & & \\
$\quad$ Baseline, with habitual drops & 25 & $36.8 \pm 26.9$ & \\
$\quad$ After 4 weeks with study drops & 22 & $29.1 \pm 24.3$ & \\
$\quad$ Change from baseline & 22 & $-5.0 \pm 20.4$ & 0.260 \\
Overall productivity loss, \% & & & \\
$\quad$ Baseline, with habitual drops & 22 & $36.3 \pm 26.4$ & \\
$\quad$ After 4 weeks with study drops & 19 & $22.7 \pm 18.3$ & \\
$\quad$ Change from baseline & $18^{\mathrm{a}}$ & $-7.2 \pm 19.4$ & 0.140 \\
Non-work activity, \% impairment & & & \\
Baseline, with habitual drops & 48 & $40.0 \pm 27.1$ & \\
After 4 weeks with study drops & 46 & $27.6 \pm 25.1$ & \\
Change from baseline & $45^{\mathrm{a}}$ & $-11.3 \pm 26.4$ & 0.006 \\
\hline
\end{tabular}

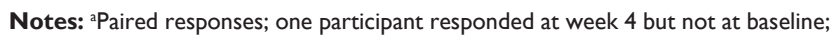
bfor participants who provided data for both presenteeism and absenteeism. Abbreviation: SD, standard deviation. event was blurred or blurry vision (3/49 patients, 6.1\%), which occurred after instillation of the study medication. One patient reported redness of the eyes, one reported stinging and burning due to the study drops, and another reported an increase in anxiety.

\section{Discussion}

The participants in this study had mild to moderate MGD, as evidenced by their mean gland expression grades (their meibum was opaque and turbid with normal or increased viscosity, instead of normal clear oil) and by their mean gland dropout (patients were missing $5.5 \pm 4.3$ glands per eye, out of an expected normal number of $\sim 23$ glands per eye) ${ }^{24}$ For the 48 patients with MGD who completed a 1-week lead-in period with their habitual eye drops and then a 4-week treatment period with Systane Balance, the most notable outcomes were for the subjective effects rather than objective signs of MGD. For example, the results on the validated IDEEL questionnaire indicated that patient-reported improvements after treatment with study medication were statistically and clinically significant. These outcomes were achieved after the patients dosed the study medication significantly less frequently than they had dosed their habitual medication. Along with these improvements in subjective symptoms and related lifestyle effects, some improvements in the signs of MGD were observed, such as statistically significant but clinically modest improvements in corneal staining and tear film breakup time. 
Table 4 Patient assessments of study medication after 4 weeks of treatment

\begin{tabular}{|c|c|c|c|c|c|}
\hline Responses, n (\%) & $\begin{array}{l}\text { Strongly } \\
\text { disagree }\end{array}$ & Disagree & Undecided & Agree & $\begin{array}{l}\text { Strongly } \\
\text { agree }\end{array}$ \\
\hline Study drops successfully treated dry eye symptoms & $3(6.4)$ & $6(12.8)$ & $8(17.0)$ & $18(38.3)$ & $12(25.5)$ \\
\hline Study drops are soothing & $4(8.5)$ & $4(8.5)$ & $8(17.0)$ & $17(36.2)$ & $14(29.8)$ \\
\hline Study drops are moisturizing & $2(4.3)$ & $7(14.9)$ & $4(8.5)$ & $19(40.4)$ & $15(31.9)$ \\
\hline Would continue to use study drops & $4(8.5)$ & $6(12.8)$ & $7(14.9)$ & $12(25.5)$ & $18(38.3)$ \\
\hline Study drops are more effective than previous drops & $4(8.5)$ & $8(17.0)$ & $10(2 \mid .3)$ & $12(25.5)$ & $13(27.7)$ \\
\hline Study drops work faster than previous drops & $3(6.4)$ & $8(17.0)$ & $9(19.1)$ & $14(29.8)$ & $13(27.7)$ \\
\hline Dry eye symptoms are much better now & $2(4.3)$ & $9(19.1)$ & $5(10.6)$ & $20(42.6)$ & II (23.4) \\
\hline
\end{tabular}

Note: For each question, $n=47$ respondents.

Specific quality-of-life measures that were significantly improved after treatment with the study medication were scores for the IDEEL subscales of Daily Activity Limitations, Emotional Well-Being, and Work Limitations and the WPAI:SHP subscale of Non-Work Activity Impairment (for activities such as housework, exercising, and studying). No significant quality-of-life improvement was observed on the WPAI:SHP Work Activity subscale, but fewer than half of the study participants held employment during the study, limiting the usefulness of that subscale. Moreover, mild to moderate MGD is presumed a low-grade and bothersome dysfunction, but not a work-prohibiting problem. The non-work-related quality-of-life improvements found in this study may be partially related to the significantly reduced need to dose with eye drops (study medication versus habitual therapy); dosing less frequently means fewer interruptions to lifestyle. The reduced need to dose with the study drops indicated a sustained effect of the medication; indeed, research has shown that tear film breakup time is significantly longer 2 hours after dosing with Systane Balance than with comparator emollient lubricant eye drops. ${ }^{25}$

In addition to the general quality-of-life improvements, specific symptom alleviation was indicated by the clinically and statistically significant improvements observed on the IDEEL Symptom Bother subscale/module. The mean 14-point improvement (on a scale of 0 to 100) in this study represented a moderate effect size and exceeded the previously established threshold ${ }^{16}$ of twelve points for a minimum clinically important difference. The symptom improvement was likely mediated through direct action of the eye drops, and may also have been mediated through indirect action of the eye drops. Directly, the study medication has been shown to produce a tear film lipid layer significantly thicker than that produced by comparator emollient eye drops for at least an hour after dosing. ${ }^{25}$ Lipid layer thickness correlates directly to symptom scores. ${ }^{9}$ Indirectly, the study medication may have alleviated the clogging of the meibomian glands over the course of the 4 weeks of treatment (since the drops contain oily excipients and like dissolves like), resulting in gland expression grades that improved modestly but statistically significantly. More investigation into this gland expression finding is needed, using meibography methods as previously described ${ }^{26-28}$ (instead of simple clinical grading at the slit lamp).

Patients' perceptions of the therapy that achieved these effects were generally positive. For the primary study endpoint of patients' treatment preference, $61.4 \%$ (27/44) of patients responded that they would choose the new study medication over their previous medication. A majority of patients ( $>53 \%$ ) had favorable impressions of, and satisfaction with, the study eye drops (on the other ten questions on the preference survey). On the IDEEL subscales, treatment bother was significantly lessened (with a moderate effect size) and treatment satisfaction was significantly improved (with a moderate effect size). The most commonly reported treatment-related adverse event was blurred or blurry vision (3/49 patients, $6.1 \%$ ), occurring after instillation of the study medication. Some blurring was expected, as lipid-containing lubricant drops as a class induce blurring after instillation

Table 5 Patient satisfaction with study medication after 4 weeks of treatment

\begin{tabular}{|c|c|c|c|c|c|}
\hline Responses, n (\%) & $\begin{array}{l}\text { Very } \\
\text { dissatisfied }\end{array}$ & $\begin{array}{l}\text { Somewhat } \\
\text { dissatisfied }\end{array}$ & Undecided & $\begin{array}{l}\text { Somewhat } \\
\text { satisfied }\end{array}$ & $\begin{array}{l}\text { Very } \\
\text { satisfied }\end{array}$ \\
\hline Satisfaction with how quickly the study eye drops worked & $2(4.3)$ & $3(6.4)$ & $2(4.3)$ & $20(42.6)$ & $20(42.6)$ \\
\hline Satisfaction with the comfort of the eye drops & $3(6.4)$ & $5(10.6)$ & $2(4.3)$ & $15(31.9)$ & $22(46.8)$ \\
\hline Satisfaction with the eye drops overall & $3(6.4)$ & $5(10.6)$ & $2(4.3)$ & $20(42.6)$ & $17(36.2)$ \\
\hline
\end{tabular}

Note: For each question, $n=47$ respondents. 


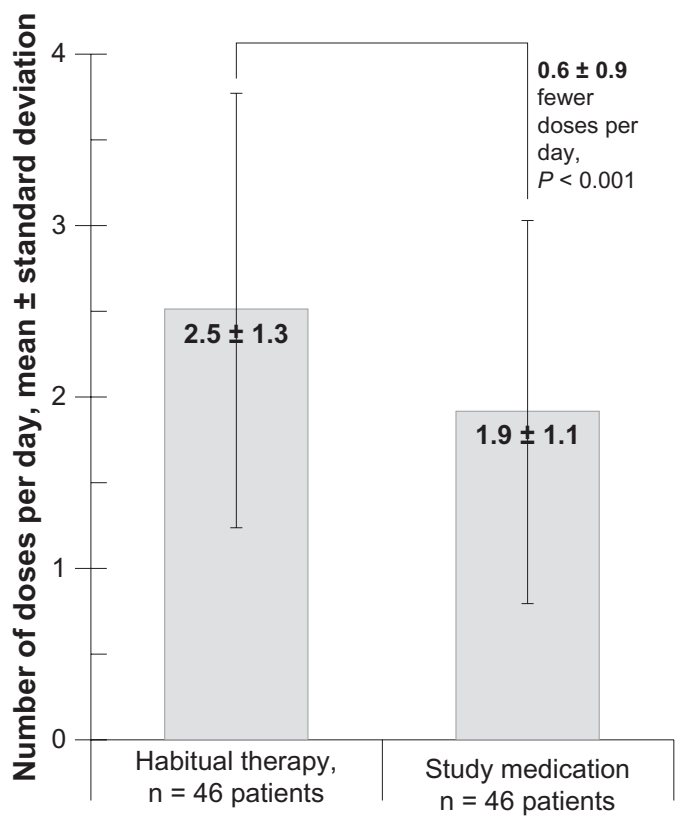

Figure 2 Dosing frequency for the 46 study participants whose electronic dosage records were complete at both time points (after I week of habitual therapy and after 4 weeks of study medication).

(though newer formulations have been better accepted by patients $)^{10}$ and blurring is expected any time the tear film is disrupted or changed, regardless of the formulation of the eye drops that induces the change. For example, blurred vision was reported as an adverse event in both arms of a study of Systane Ultra Lubricant Eye Drops (Alcon) versus Refresh $^{\circledR}$ Optive ${ }^{\mathrm{TM}}$ Lubricant Eye Drops (Allergan, Irvine, CA, USA). ${ }^{29}$

Effects of the therapy were also perceptible to the physicians. Tear film breakup time and corneal staining improved at magnitudes that were clinically modest but statistically significant. These improved signs of MGD are

Table 6 Tear film breakup time

\begin{tabular}{lll}
\hline & Patients, $\mathbf{n}$ & $\begin{array}{l}\text { Tear film breakup } \\
\text { time, mean } \pm \text { SD }\end{array}$ \\
\hline Replicate I & & \\
Baseline, seconds & 49 & $5.3 \pm 2.3$ \\
Visit 4, seconds & 48 & $6.0 \pm 2.2$ \\
$\quad$ Change from baseline, seconds & 48 & $0.6 \pm 2.1^{*}$ \\
$\quad$ Change from baseline, \% & 48 & $24 \pm 55$ \\
Replicate 2 & & \\
Baseline, seconds & 49 & $5.3 \pm 2.5$ \\
Visit 4, seconds & 47 & $6.0 \pm 2.3$ \\
$\quad$ Change from baseline, seconds & 47 & $0.6 \pm 2 . I^{*}$ \\
$\quad$ Change from baseline, \% & 47 & $21 \pm 45$ \\
\hline
\end{tabular}

Notes: Values were averaged from both eyes of each patient. $* P<0.05$.

Abbreviation: SD, standard deviation.
Table 7 Corneal staining

\begin{tabular}{|c|c|c|}
\hline & \multicolumn{2}{|c|}{ Mean \pm standard deviation } \\
\hline & $\begin{array}{l}\text { Baseline, } \\
n=49\end{array}$ & $\begin{array}{l}\text { Visit 4, } \\
n=47\end{array}$ \\
\hline \multicolumn{3}{|c|}{ Central cornea } \\
\hline Score & $0.5 \pm 0.6$ & $0.3 \pm 0.4$ \\
\hline Improvement & & $-0.2 \pm 0.5^{*}$ \\
\hline \multicolumn{3}{|c|}{ Inferior cornea } \\
\hline Score ${ }^{a}$ & $1.3 \pm 0.7$ & $\mathrm{I} . \mathrm{I} \pm 0.8$ \\
\hline Improvement & & $-0.3 \pm 0.5^{*}$ \\
\hline \multicolumn{3}{|l|}{ Nasal cornea } \\
\hline Score ${ }^{a}$ & $1.0 \pm 0.7$ & $0.8 \pm 0.7$ \\
\hline Improvement & & $-0.2 \pm 0.6^{*}$ \\
\hline \multicolumn{3}{|c|}{ Superior cornea } \\
\hline Score & $0.5 \pm 0.5$ & $0.4 \pm 0.5$ \\
\hline Improvement & & $-0.2 \pm 0.4^{*}$ \\
\hline \multicolumn{3}{|c|}{ Temporal cornea } \\
\hline Score & $0.8 \pm 0.6$ & $0.5 \pm 0.5$ \\
\hline Improvement & & $-0.2 \pm 0.6^{*}$ \\
\hline \multicolumn{3}{|l|}{ Total score } \\
\hline Score ${ }^{b}$ & $4.1 \pm 2.3$ & $3.1 \pm 2.1$ \\
\hline Improvement & & $-1.0 \pm 1.3^{* *}$ \\
\hline
\end{tabular}

Notes: Values were averaged from both eyes of each patient. ${ }^{2}$ On a scale where 0 = none, $\mathrm{I}=$ mild, 2 = moderate, and 3 = severe; 'bum of the five corneal sectors, yielding a scale from 0 to $15 . * P<0.05 ; * P<0.001$.

compared to similar outcomes from a study ${ }^{30}$ of patients treated with cyclosporine, $0.5 \%$ or artificial tears (similar to the habitual therapies in the current study) in Table 8 .

\section{Limitations and future directions}

Limitations of this study include the unmasked design (which may have allowed brand loyalties to affect the outcomes), lack of a comparator group, short follow-up duration, and limited study population. The limited study population also precluded post-hoc investigation of differences between premenopausal versus postmenopausal ages or between sexes, though it should be noted that the study population was $85.7 \%$ (42/49) female. Double-masked studies are needed, using active controls to allow comparisons, and using combination therapies to mimic real-world situations more closely. Studies should also be stratified by type and severity of MGD, since MGD is a heterogenous group of disorders; the current study demonstrated that mild/moderate MGD was alleviated, but it would be interesting to see which types of MGD patients could benefit most from these eye drops. Finally, while this study assessed the lipid component of the tears of the patients, an assessment of the overall production of tears (via Schirmer's test) would have been informative but was not necessary for diagnosis of evaporative dry eye due to MGD. 
Table 8 Comparison of signs of meibomian gland dysfunction in two studies of patients treated with topical cyclosporine, artificial tears, or Systane ${ }^{\circledR}$ Balance Lubricant Eye Drops (Alcon Inc, Fort Worth, TX, USA)

\begin{tabular}{|c|c|c|c|c|c|}
\hline & \multirow[t]{2}{*}{$\begin{array}{l}\text { Patients at } \\
\text { endpoint, } n\end{array}$} & \multicolumn{2}{|c|}{$\begin{array}{l}\text { Tear film breakup time, }{ }^{a} \\
\text { mean } \pm \text { SD, seconds }\end{array}$} & \multicolumn{2}{|c|}{$\begin{array}{l}\text { Corneal staining, } \\
\text { mean } \pm \text { SD, units }\end{array}$} \\
\hline & & Baseline & Endpoint $^{c}$ & Baseline & Endpoint $^{c}$ \\
\hline \multicolumn{6}{|l|}{ Current study } \\
\hline $\begin{array}{l}\text { Systane Balance, } \\
\text { I month }\end{array}$ & 48 & $5.3 \pm 2.3$ & $\begin{array}{l}6.0 \pm 2.2 \\
(P<0.05)\end{array}$ & $4.1 \pm 2.3$ & $\begin{array}{l}3.1 \pm 2.1 \\
(P<0.00 I)\end{array}$ \\
\hline \multicolumn{6}{|l|}{ Reference study ${ }^{30}$} \\
\hline $\begin{array}{l}\text { Cyclosporine, } 0.5 \% \text {, } \\
3 \text { months }\end{array}$ & 12 & $9.9 \pm 10.3$ & $\begin{array}{l}10.8 \pm 8.0 \\
(P=0.08)\end{array}$ & $3.3 \pm 2.4$ & $\begin{array}{l}1.3 \pm 1.9 \\
(P=0.01)\end{array}$ \\
\hline $\begin{array}{l}\text { Artificial tears, } \\
3 \text { months }\end{array}$ & 14 & $7.4 \pm 6.6$ & $\begin{array}{l}6.1 \pm 3.8 \\
(P=0.08)\end{array}$ & $4.4 \pm 3.0$ & $\begin{array}{l}3.9 \pm 2.9 \\
(P=0.01)\end{array}$ \\
\hline
\end{tabular}

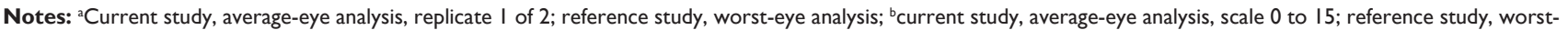
eye analysis, scale 0 to 12; 'current study, significance versus baseline; reference study, significance versus comparator arm.

Abbreviation: SD, standard deviation.

\section{Conclusion}

The study reported here demonstrated that a novel artificial tear emulsion, Systane Balance Lubricant Eye Drops, was effective in controlling both the signs and the symptoms of dry eye in MGD patients. Improved quality-of-life outcomes and reduced dosing frequency (relative to values with habitual therapy) occurred with Systane Balance after only 4 weeks of use by MGD patients.

\section{Disclosure}

Alcon Research funded this study and provided the assistance of a medical writer. Dr Sindt has received research grants from Alcon Vision Care and is a consultant for Alcon and Vistakon. Dr Foulks is currently or has been in a consultancy relationship with Bausch and Lomb, Eleven Biotherapeutics, R Tech Ueno, Santen, and Alcon.

\section{References}

1. The definition and classification of dry eye disease: report of the Definition and Classification Subcommittee of the International Dry Eye WorkShop (2007). Ocul Surf. 2007;5(2):75-92.

2. Nelson JD, Shimazaki J, Benitez-del-Castillo JM, et al. The International Workshop on Meibomian Gland Dysfunction: Report of the Definition and Classification Subcommittee. Invest Ophthalmol Vis Sci. 2011;52(4):1930-1937.

3. Albietz JM. Prevalence of dry eye subtypes in clinical optometry practice. Optom Vis Sci. 2000;77(7):357-363.

4. Lekhanont K, Rojanaporn D, Chuck RS, Vongthongsri A. Prevalence of dry eye in Bangkok, Thailand. Cornea. 2006;25(10):1162-1167.

5. Lin PY, Tsai SY, Cheng CY, Liu JH, Chou P, Hsu WM. Prevalence of dry eye among an elderly Chinese population in Taiwan: the Shihpai Eye Study. Ophthalmology. 2003;110(6):1096-1101.

6. Nichols KK. The international workshop on meibomian gland dysfunction: introduction. Invest Ophthalmol Vis Sci. 2011;52(4): 1917-1921.

7. Nichols KK, Foulks GN, Bron AJ, et al. The international workshop on meibomian gland dysfunction: executive summary. Invest Ophthalmol Vis Sci. 2011;52(4):1922-1929.
8. Nichols KK, Nichols JJ, Mitchell GL. The lack of association between signs and symptoms in patients with dry eye disease. Cornea. 2004;23(8):762-770.

9. Isreb MA, Greiner JV, Korb DR, et al. Correlation of lipid layer thickness measurements with fluorescein tear film break-up time and Schirmer's test. Eye. 2003;17(1):79-83.

10. Geerling G, Tauber J, Baudouin C, et al. The international workshop on meibomian gland dysfunction: report of the subcommittee on management and treatment of meibomian gland dysfunction. Invest Ophthalmol Vis Sci. 2011;52(4):2050-2064.

11. Benelli U. Systane lubricant eye drops in the management of ocular dryness. Clin Ophthalmol. 2011;5:783-790.

12. Alcon Research. Evaluation of FID 114657 in dry eye subjects. In: ClinicalTrials.gov [website on the Internet]. Bethseda, MD: US National Library of Medicine; 2010 [updated January 31, 2012]. Available from: http://www.clinicaltrials.gov/ct2/show/NCT01079858. NLM identifier: NCT01079858. Accessed March 28, 2013.

13. Mathers WD, Shields WJ, Sachdev MS, Petroll WM, Jester JV. Meibomian gland dysfunction in chronic blepharitis. Cornea. 1991;10(4):277-285.

14. Lemp MA. Report of the National Eye Institute/Industry workshop on Clinical Trials in Dry Eyes. CLAO J. 1995;21(4):221-232.

15. Rajagopalan K, Abetz L, Mertzanis P, et al. Comparing the discriminative validity of two generic and one disease-specific health-related quality of life measures in a sample of patients with dry eye. Value Health. 2003;8(2):168-174.

16. Fairchild CJ, Chalmers RL, Begley CG. Clinically important difference in dry eye: change in IDEEL-symptom bother. Optom Vis Sci. 2008;85(8):699-707.

17. Reilly MC, Zbrozek AS, Dukes EM. The validity and reproducibility of a work productivity and activity impairment instrument. Pharmacoeconomics. 1993;4(5):353-365.

18. Reilly MC, Gerlier L, Brabant Y, Brown M. Validity, reliability, and responsiveness of the work productivity and activity impairment questionnaire in Crohn's disease. Clin Ther. 2008;30(2):393-404.

19. Yu J, Asche CV, Fairchild CJ. The economic burden of dry eye disease in the United States: a decision tree analysis. Cornea. 2011;30(4): 379-387.

20. Shi L, Liu J, Koleva Y, Fonseca V, Kalsekar A, Pawaskar M. Concordance of adherence measurement using self-reported adherence questionnaires and medication monitoring devices. Pharmacoeconomics. 2010;28(12):1097-1107.

21. Robin AL, Novack GD, Covert DW, Crockett RS, Marcic TS. Adherence in glaucoma: objective measurements of once-daily and adjunctive medication use. Am J Ophthalmol. 2007;144(4):533-540. 
22. Siatkowski RM, Cotter S, Miller JM, et al; US Pirenzepine Study Group. Safety and efficacy of $2 \%$ pirenzepine ophthalmic gel in children with myopia: a 1-year, multicenter, double-masked, placebo-controlled parallel study. Arch Ophthalmol. 2004;122(11):1667-1674.

23. Cohen J. Statistical Power Analysis for the Behavioral Sciences, 2nd ed. Hillsdale, NJ: Lawrence Erlbaum; 1988.

24. Norn M. Meibomian orifices and Marx's line. Studied by triple vital staining. Acta Ophthalmol (Copenh). 1985;63(6):698-700.

25. Korb DR, Blackie C, Meadows D, Christensen M, Tudor M. Evaluation of extended tear stability by two emulsion-based artificial tears. Poster presented at the 6th International Conference on the Tear Film and Ocular Surface: Basic Science and Clinical Relevance, September 22-25, 2010, Florence, Italy.

26. Arita R, Itoh K, Inoue K, Amano S. Noncontact infrared meibography to document age-related changes of the meibomian glands in a normal population. Ophthalmology. 2008;115(5):911-915.
27. Arita R, Itoh $\mathrm{K}$, Maeda S, et al. Proposed diagnostic criteria for obstructive meibomian gland dysfunction. Ophthalmology. 2009;116(11): 2058-2063.

28. Arita R, Itoh K, Maeda S, Maeda K, Amano S. A newly developed noninvasive and mobile pen-shaped meibography system. Cornea. 2013;32(3):242-247.

29. Davitt WF, Bloomenstein M, Christensen M, Martin AE. Efficacy in patients with dry eye after treatment with a new lubricant eye drop formulation. J Ocul Pharmacol Ther. 2010;26(4):347-353.

30. Perry HD, Doshi-Carnevale S, Donnenfeld ED, Solomon R, Biser SA, Bloom AH. Efficacy of commercially available topical cyclosporine A $0.05 \%$ in the treatment of meibomian gland dysfunction. Cornea. 2006;25(2):171-175.
Clinical Ophthalmology

\section{Publish your work in this journal}

Clinical Ophthalmology is an international, peer-reviewed journal covering all subspecialties within ophthalmology. Key topics include: Optometry; Visual science; Pharmacology and drug therapy in eye diseases; Basic Sciences; Primary and Secondary eye care; Patient Safety and Quality of Care Improvements. This journal is indexed on Submit your manuscript here: http://www.dovepress.com/clinical-ophthalmology-journal

\section{Dovepress}

PubMed Central and CAS, and is the official journal of The Society of Clinical Ophthalmology (SCO). The manuscript management system is completely online and includes a very quick and fair peer-review system, which is all easy to use. Visit http://www.dovepress.com/ testimonials.php to read real quotes from published authors. 